\title{
e-Migrinter
}

22 | 2021

Varia

\section{« Filmer les migrations »}

Compte-rendu de la $5^{\mathrm{e}}$ séance du séminaire « Les représentations des migrations » organisé par Migrinter, le 18 mai 2021

\section{Laura Bouillette et Flora Penot}

\section{OpenEdition}

Journals

Édition électronique

URL : https://journals.openedition.org/e-migrinter/2759

DOI : $10.4000 /$ e-migrinter.2759

ISSN : 1961-9685

Éditeur

UMR 7301 - Migrinter

Référence électronique

Laura Bouillette et Flora Penot, « «Filmer les migrations » », e-Migrinter [En ligne], 22 | 2021, mis en ligne le 25 novembre 2021, consulté le 17 décembre 2021. URL : http://journals.openedition.org/emigrinter/2759; DOI : https://doi.org/10.4000/e-migrinter.2759

Ce document a été généré automatiquement le 17 décembre 2021.

Tous droits réservés 


\section{«Filmer les migrations »}

Compte-rendu de la $5^{\mathrm{e}}$ séance du séminaire « Les représentations des migrations » organisé par Migrinter, le 18 mai 2021

\section{Laura Bouillette et Flora Penot}

\section{RÉFÉRENCE}

« Filmer les migrations ». $5^{\mathrm{e}}$ séance du séminaire "Les représentations des migrations » organisé par Migrinter

1 La séance de séminaire "Filmer les migrations ", du mardi 18 mai 2021, avait pour invités deux chercheurs-réalisateurs, Elsa Gomis et Benoît Raoulx, et s'est inscrite dans le cadre du séminaire du laboratoire Migrinter intitulé «Les représentations des migrations ", mis en place depuis 2019. Les précédentes séances ont abordé différents supports qui permettent d'interroger les représentations des migrations telles que la cartographie, la bande dessinée et la photographie témoignant de : "l'évolution historique des représentations matérielles des migrations, la place du migrant ou de la migrante dans ce processus de mise en images, ou encore la co-construction de ces représentations entre monde scientifique et artistique. $»^{1}$. Cette séance de séminaire propose ainsi une réflexion sur la mise en représentation de l'espace migratoire et des mouvements de ces personnes par le biais de l'image animée, ici sous la forme de documentaires. Les deux intervenants ont développé à la fois le processus de création filmique, la question des différents plans et prises de vue, ainsi que leurs sources et inspirations artistiques.

\section{L'Encyclopédie des migrants, documentaire réalisé par Frédéric Leterrier et Benoit Raoulx (2017)}

2 Benoît Raoulx est maître de conférences HDR au sein de l'Université de CaenNormandie et responsable du pôle "Filmer la recherche en sciences humaines" (FRESH) au sein de la MSHB ${ }^{2}$ Rennes, MRSH Caen et du Centre anthropologique de 
Sousse (Tunisie). Ses recherches en géographie sociale et culturelle portent essentiellement sur l'étude de la marginalité spatiale et la marginalité sociale. C'est en tant que co-réalisateur, aux côtés de Frédéric Leterrier, du documentaire " L'encyclopédie des migrants " qu'il est intervenu lors de la séance. Sorti en 2017, ce court-métrage a pour objectif de mettre en lumière le processus de réalisation et de coordination du projet homonyme en nous emmenant au plus près des 700 acteurs $\mathrm{y}$ ayant contribué.

3 «L'encyclopédie des migrants » est un projet artistique à l'initiative de l'artiste Paloma Fernandez Sobrino et coordonné par l'association rennaise L'âge de la tortue ${ }^{3}$. Ce projet s'est déroulé entre 2014 et 2017 dans huit villes se trouvant sur la façade Atlantique entre la Bretagne et Gibraltar. Cette encyclopédie regroupe 400 témoignages de personnes migrantes restitués sous forme de lettres manuscrites, chacune agrémentée d'un portrait photo de son écrivain. On peut également y lire 16 textes rédigés par des chercheurs en sciences humaines et sociales. Malgré l'espace géographique vaste abordé dans le reportage, les huit villes réparties dans quatre pays (France, Espagne, Portugal et Gibraltar) ne sont que des personnages secondaires de l'histoire racontée. Ce sont les personnes et les langages qui sont au centre du projet. Chaque témoin est invité à écrire une lettre manuscrite à l'attention d'un proche se trouvant loin et dans laquelle l'auteur exprime comment cette distance l'affecte. Pour Paloma Fernandez Sobrinot, parler de solitude et d'intimité à travers les lettres est une approche différente et éloignée de celle des discours politiques et médiatiques.

4 Plusieurs aspects du projet sont abordés dans le documentaire avec des mises en scène distinctes: les réunions multilingues (français, espagnol, anglais) entre chercheurs venus des huit villes du projet ainsi que les moments entre photographes et témoins, sont filmés sans mise en situation particulière. Il s'agit d'une prise de vue directe avec une position extérieure et neutre du réalisateur. C'est selon un autre dispositif filmique que les moments de restitution orale des lettres se déroulent avec un plan serré sur le visage du lecteur. Les entretiens avec les personnes travaillant sur le projet et ceux avec les témoins qui y ont participé, sont eux, filmés sur fond noir. Ces choix permettent de mettre en place une bulle de la parole, qui offre une profondeur plus importante, une fenêtre sur le ressenti et sur l'intimité des témoins.

Des « respirations » sont inclues dans ce documentaire par le biais de prises de vues de l'océan. Il s'agit de plans fixes entre les phases d'entretien intime offrant une proximité entre interviewé et spectateur, et des instants de collégialité entre les chercheurs auxquels le spectateur reste extérieur. Ces plans méditatifs sont d'autant plus marquants par l'absence de voix off. Ainsi, les sous-titres prennent un rôle central, non seulement pour la traduction des nombreuses langues parlées (français, espagnol, anglais, portugais) mais également pour des éléments non abordés directement dans le documentaire tels que la présentation du projet au début du film.

6 L'aspect collaboratif entre toutes les équipes impliquées dans le projet permet de mettre en lumière les divergences d'opinion et les compromis devant être faits pour une œuvre de si grande envergure. Le spectateur assiste aux discussions et désaccords sur les limitations d'expressions imposées par l'équipe de Rennes, qui pour l'équipe de Gibraltar va à l'encontre de l'objectif même du projet (limitation de la longueur des lettres manuscrites, cadre trop strict pour les chercheurs : deux pages recto verso sans note de bas de pages ou de références, etc.). Ce documentaire offre aux spectateurs 
différentes façons d'appréhender le processus de co-construction du projet à travers la retranscription fidèle du regard porté par les acteurs sur leur participation.

\section{The people behind the scenes, documentaire réalisé par Elsa Gomis (2019)}

Elsa Gomis est une réalisatrice et chercheuse en anthropologie de l'image à l'Université d'East Anglia, à Norwich en Angleterre. Ses travaux se situent au croisement des arts plastiques et des études migratoires, et contribuent à interroger les images dominantes qui structurent l'imaginaire de la migration. Elle co-dirige également depuis 2019, la section « images » du journal De Facto de l'Institut Convergences Migrations, à Paris.

Dans le cadre de son doctorat, elle a réalisé un documentaire intitulé The people behind the scenes. Essai filmique sur l'imaginaire collectif des migrations (2019) ayant pour décor l'archipel de Malte, représentant une véritable caisse de résonance des politiques migratoires européennes actuelles. Ce long-métrage aborde l'exil et les naufrages en évoquant indirectement les migrants noyés dans la Méditerranée, s'éloignant ainsi des images dominantes diffusées par les médias de cette "crise » migratoire. A travers différents entretiens, Elsa Gomis fait le choix de donner la parole aux maltais en évoquant avec eux différentes représentations des migrations au cours de leur vie et de l'histoire de l'archipel. L'approche biographique avec l'apport d'images personnelles, ajoute une profondeur à cette lecture différente de l'actualité migratoire en Méditerranée. Cette œuvre dévoile l'apport épistémologique de l'outil filmique dans le champ des études migratoires.

La réalisatrice interpelle le spectateur dès le début de son film, en annonçant la place et le rôle que peuvent avoir les prises de vue : Les images font plus que nous refléter, elles parlent de nos relations aux autres. Elle souhaite comprendre ce que les images veulent dire de l'Autre, de l'étranger qui vient, en cheminant par la mer. Il s'agit alors d'évoquer un sentiment commun de l'exil dans ce film. En puisant dans ses archives familiales, Elsa Gomis était à la recherche d'images déjà présentes dans ses souvenirs afin que sa démarche individuelle - les images tournées par son grand-père - prenne ensuite une dimension universelle avec les séquences actuelles prises à Malte. Cette démarche souhaite réinitialiser les imaginaires migratoires, en opposition aux discours et images dominants des médias, qui ne cessent d'insister sur la spectacularité de la frontière et le côté misérabiliste des traversées en Méditerranée.

Selon Elsa Gomis, les personnes migrantes ne se sentent plus représentées par les images mais plutôt exclues par elles à travers différents stéréotypes. Le problème des stéréotypes ne signifie pas une réalité fausse mais incomplète, qui ne rend pas compte de la complexité des profils des migrants venus par la mer. La Méditerranée est en effet la région la plus surveillée au monde et la plus meurtrière. Tout au long de son œuvre, la réalisatrice dessine un sentiment de l'exil et s'inscrit dans la sphère de l'art contemporain avec une polysémie des motifs liés à la mer et aux naufrages (épaves de bateaux, pneumatiques oranges/jaunes, bateaux échoués, barques traditionnelles de pêcheurs, ...). Ses différentes prises de vue de l'archipel maltais apportent plusieurs métaphores (gilets de sauvetage tels des corps échoués), des accumulations avec des emballages (gilets de sauvetage, morceaux de plastique) et donne une impression de survivance d'images passées. Les images dévoilent ainsi une dimension anachronique 
dans son film et permettent de naviguer d'une époque à l'autre, entre les archives familiales reconnaissables au " grain » des anciennes pellicules et les prises de vue maltaises tournées en 2018.

11 Elsa Gomis définit l'imaginaire de l'exil comme étant un ensemble composé de matériaux, de récits et de motifs qui s'écoulent au fil du temps et des acteurs rencontrés. Ses propos au début du film apportent un éclairage sur la façon dont elle perçoit le film comme un outil heuristique, qui donne à voir et à comprendre. Ce longmétrage se structure ainsi en huit grandes étapes, mentionnées en anglais lors du visionnage et dévoilent une certaine chronologie : " Knowing the history », " Being careful », « Listening to a Fabulous story ", « Monitor », « To fly », « To count », « To break the mirror ", " From my images of exile I will remember these ones ». Alternant entre entretiens portant sur la vie quotidienne de l'île (habitants, touristes, chercheurs) et prises de vue à la manière du documentaire, les voix off de la réalisatrice et des enquêtés se succèdent sur différents thèmes : l'histoire des grands tournages du cinéma à Malte (blockbusters américains), le tourisme et le marketing territorial de l'archipel (spots publicitaires), le rapport à la mer et à la mémoire des marins avec l'Odyssée d'Homère selon un chercheur en archéologie marine, etc.

Le processus de création de ce long-métrage se base enfin sur un écrit, rédigé au début de sa thèse. Le film en tant que support artistique constitue un déclencheur pour l'entretien puisque Elsa Gomis montre des images, susceptibles d'être interprétées de différentes manières, pour interroger les personnes enquêtées. La prise d'images a été menée par la réalisatrice elle-même, à l'aide d'un cadreur. Elle est pleinement directrice de son œuvre en étant à l'origine du montage, des sous-titrages et de la traduction établis au sein des locaux de l'École Louis Lumière. Bénéficiant d'un petit budget, elle a associé le son et l'image en traitant des dizaines d'heures de rush, et a comptabilisé au cours de son enquête, une quarantaine d'entretiens récoltés en cinq semaines.

\section{Conclusion}

13 Les deux documentaires présentés lors de cette séance permettent d'accéder à un autre angle de vue sur les migrations que celui habituellement abordé. Dans L'encyclopédie des migrants, ce sont les coulisses d'une recherche qui sont explorées par le biais de témoignages reconstruisant le processus de création de ce projet faramineux alors que dans The people behind the scene, c'est à travers une histoire familiale agrémentée de récits d'expériences des maltais que le contexte migratoire méditerranéen est reconstitué.

14 Le visionnage des documentaires, et cette séance de séminaire " Filmer les migrations » présentent ainsi des informations et une compréhension des événements qu'un écrit seul ne permet pas. En effet, l'outil filmique offre une multitude d'opportunités, d'approfondissement des discours et des récits des personnes migrantes en donnant, entre-autre, un accès aux émotions de l'interlocuteur. Cette démarche oblige ainsi à repositionner le regard sur les personnes en exil et plus généralement sur la migration. 


\section{NOTES}

1. http://migrinter.labo.univ-poitiers.fr/les-representations-des-migrations-seance5/

2. Maison des Sciences de l'Homme en Bretagne

3. L'âge de la Tortue est une association mettant en œuvre des projets artistiques basés sur une réflexion critique de notre société contemporaine et le respect des droits culturels.

\section{AUTEURS}

\section{LAURA BOUILLETTE}

Doctorante en géographie

Laboratoire Migrinter - Université de Poitiers

\section{FLORA PENOT}

Doctorante en géographie

Laboratoire Migrinter - Université de Poitiers 